\title{
A novel method for reconstructing the sinus and annulus for the treatment of annuloaortic ectasia
}

\author{
Takeshi Shimamoto, MD, ${ }^{\mathrm{a}}$ Akira Marui, MD, PhD, ${ }^{\mathrm{a}}$ Takeshi Nishina, MD, PhD, ${ }^{\mathrm{a}}$ Yoshiaki Saji, MD, ${ }^{\mathrm{a}}$ and \\ Masashi Komeda, MD, PhD, ${ }^{\text {a,b,c }}$ Kyoto, Toyohashi, and Yamato, Japan
}

Video clip is available online.

The treatment of aortic root dilatation with a leaking valve requires a multimodal approach, including root reimplantation or remodeling with or without tailoring of the sinotubular junction and valvular apparatus. ${ }^{1,2}$ However, valve-sparing procedures are time-consuming, technically demanding, and associated with intraoperative bleeding.

Here we report a novel reconstruction method for the treatment of annuloaortic ectasia with a leaking valve. In this method the dilated sinuses were excluded, and the annulus was effectively suspended by suturing a bovine pericardial patch inside the dilated aortic root.

\section{CLINICAL SUMMARY}

A 47-year-old man was referred to our hospital for the treatment of aortic regurgitation (AR) and aortic arch aneurysm; his cardiac status was New York Heart Association class III. The coronary angiographic findings were unremarkable. Transthoracic echocardiographic analysis revealed moderate-to-severe AR with dilatation of the right and noncoronary sinuses (Figure 1, A; also see Video 1). The left ventricular (LV) end-diastolic diameter was 52 $\mathrm{mm}$, and the LV ejection fraction was $54 \%$. Computed tomographic analysis showed annuloaortic ectasia with disproportionately enlarged right and noncoronary sinuses and aneurysm of the ascending aorta and aortic arch. The diameters of the sinotubular junction, ascending aorta, and aortic arch were 42,60 , and $70 \mathrm{~mm}$, respectively.

Surgical intervention was performed through a median sternotomy with the patient undergoing cardiopulmonary

\footnotetext{
From the Department of Cardiovascular Surgery, ${ }^{\text {a }}$ Kyoto University Graduate School of Medicine, Kyoto, Japan; the Department of Cardiovascular Surgery, ${ }^{\mathrm{b}}$ Toyohashi Heart Center, Toyohashi, Japan; and the Department of Cardiovascular Surgery, ${ }^{c}$ Yamato Seiwa Hospital, Yamato, Japan.

Received for publication June 3, 2008; revisions received Aug 22, 2008; accepted for publication Sept 1, 2008; available ahead of print April 17, 2009.

Address for reprints: Takeshi Shimamoto, MD, Department of Cardiovascular Surgery, Kyoto University Graduate School of Medicine, 54 Shogoin Kawahara,

Sakyo, Kyoto 606-8507, Japan (E-mail: shimamo@kuhp.kyoto-u.ac.jp).

J Thorac Cardiovasc Surg 2009; 138:1244-6

$0022-5223 / \$ 36.00$

Copyright (c) 2009 by The American Association for Thoracic Surgery doi:10.1016/j.jtcvs.2008.09.009
}

bypass. During hypothermic circulatory arrest and retrograde cerebral perfusion, the ascending aorta was incised transversely, and the aortic arch aneurysm was replaced with a $28-\mathrm{mm}$ Dacron graft with 3 branches. Subsequently, the proximal portion of the Dacron graft was clamped, and extracorporeal circulation was reinitiated. The dilated sinuses and annulus were reconstructed, and the dislocated annulus was suspended with bovine pericardial patches (Figure 2). The aortic crossclamp time was 221 minutes, and the cardiopulmonary bypass time was 287 minutes. Postoperative echocardiographic analysis showed trivial AR with improved leaflet coaptation and sinus geometry (Figure 1, $B$; also see Video 1). The diameter of the sinus reduced from $42 \mathrm{~mm}$ preoperatively to $36 \mathrm{~mm}$ postoperatively. No blood transfusion was required perioperatively, and the patient was ambulant at discharge on postoperative day 43 . At 5 years after the operation, he is doing well, his cardiac status is New York Heart Association class I, and the AR degree and LV dimensions have remained unchanged since the operation. Computed tomographic analysis performed 5 years postoperatively showed no xenograft calcification.

\section{DISCUSSION}

In the aortic root reconstruction technique for root remodeling ${ }^{1}$ or reimplantation, ${ }^{2}$ the annulus and coronary orifices are scalloped and reattached to a Dacron graft; therefore intraoperative bleeding is a potentially serious complication. Moreover, because 3 cusps are crammed into a narrow prosthesis, close investigation and meticulous adjustment are not feasible intraoperatively. Furthermore, aortic annuluses are attached to a stiff Dacron graft in both remodeling and reimplantation; this was reported to significantly increase the stress load along the leaflet attachment line compared with that seen in compliant native sinuses.

Our novel method has several advantages over the conventional methods. First, it is technically easy, particularly when only 1 or 2 sinuses are affected. A surgeon can thoroughly investigate and accurately measure the degree of annular dilatation and dislocation and therefore can meticulously adjust the degree of annular plication and uplifting, none of which is possible in the remodeling or reimplantation technique. Second, bleeding is less likely with our technique wherein a scalloped bovine pericardial patch, which is used to reconstruct the sinus and annulus, is secured inside the aortic root, thereby eliminating the need for an additional 

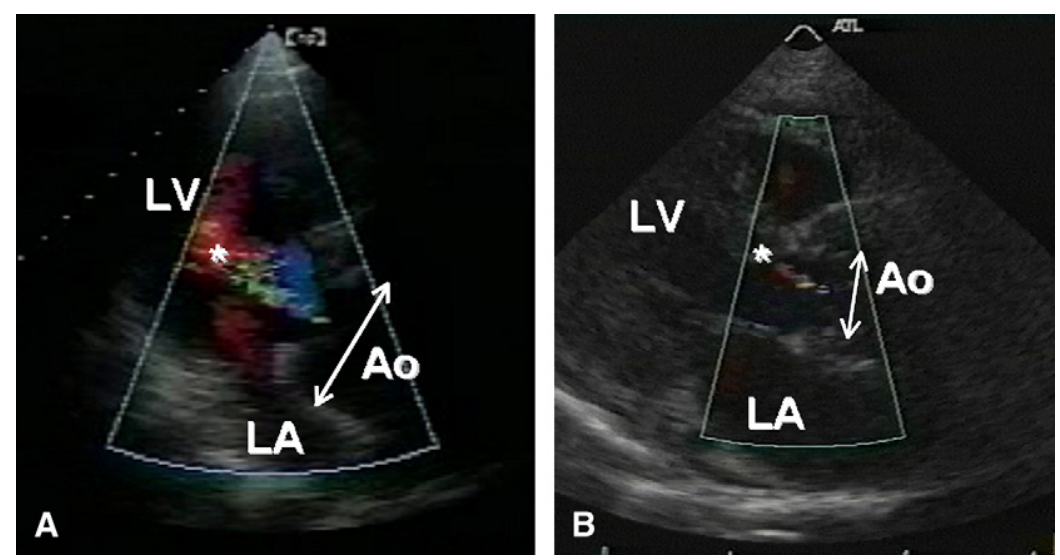

FIGURE 1. Preoperative (A) and postoperative (B) echocardiograms in an apical long-axis view showing that preoperative severe aortic regurgitation (asterisk in A) was significantly reduced to mild aortic regurgitation (asterisk in B) and that the preoperative root dilation (arrow in A) was reduced postoperatively (arrow in B). $L V$, Left ventricle; $L A$, left atrium; Ao, ascending aorta.

suture line. Third, the stress load on the leaflet attachment line is expected to be less than that in the conventional procedures because the sinus reconstructed with a soft pericardium patch is expected to be compliant.

However, our technique might have certain challenges. First, flow turbulence can occur along the patches because the reconstructed sinuses might have asymmetric shapes. In our case the turbulence could be particularly significant in the right coronary sinus because the shape of the patch was such that it was prone to distortion near the reconstructed right coronary orifice. Second, our method might not be applicable to patients with an extremely dilated annulus because in such patients a normal sinus is not available for reference. Moreover, the bovine pericardium is soft, and annular plication is mainly achieved with pledgeted sutures; therefore the degree of possible plication is intrinsically limited.
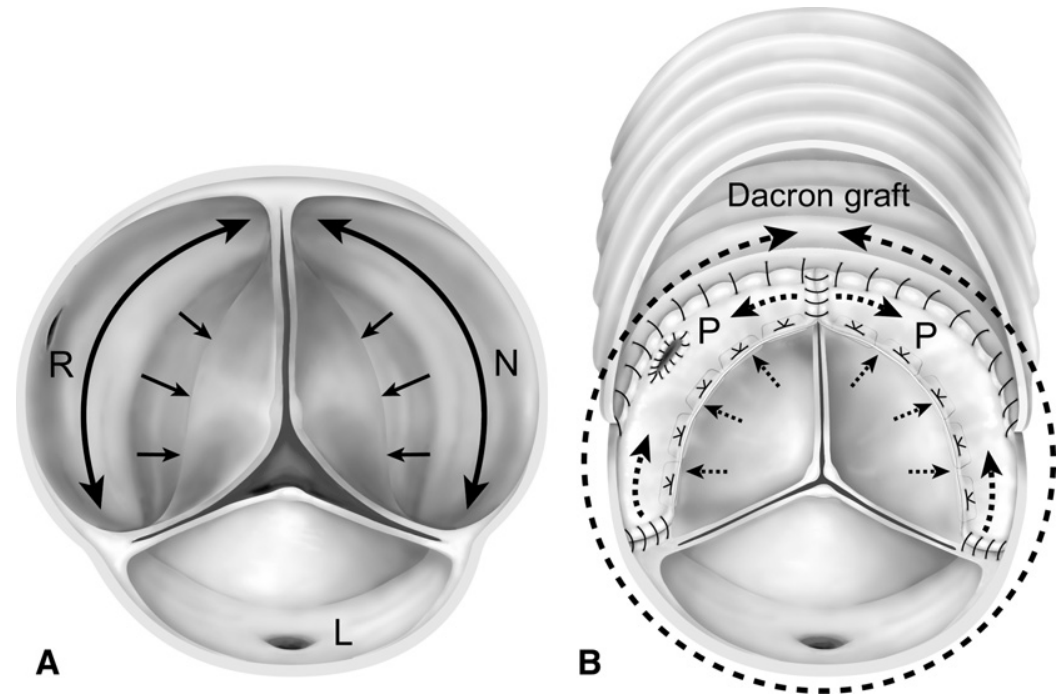

FIGURE 2. Preoperative (A) and postoperative (B) schematic presentation of the aortic root in the transaortic view. A, Marked dilatation (large arrows) and downward dislocation (small arrows) of the right and noncoronary annuluses were noticed preoperatively. The morphology of each aortic leaflet appeared near normal, and 3 commissures were of normal height. The lengths of the corresponding edges of the 3 leaflets appeared almost equivalent. B, The left coronary sinus was used as a reference because it had a normal appearance. Its length between the annulus and the coronary orifice, the annulus, and the corresponding circumferential length at the sinotubular junction were measured with silk sutures and a ruler. A bovine pericardial patch was individually scalloped according to the aforementioned measurements. The opening was tailored at the corresponding zone for the right coronary orifice. The patches were sutured to the annulus with pledgeted 5-0 polypropylene sutures, passing from the left ventricular side; this uplifted (small dotted arrows) and reduced the size of (large dotted arrows) the annulus, leading to improved leaflet coaptation. The right coronary orifice was secured to the opening of the patch with 60 polypropylene sutures. The curves of the patches were then anastomosed along the neighboring commissures up to the level of the sinotubular junction with 5-0 polypropylene sutures to uplift and plicate the aortic annulus, such that the shapes of all reconstructed sinuses were symmetric. Finally, the dilated sinotubular junction and the upper portion of the patch were anastomosed to the Dacron graft, thus achieving a reduction in the size of the sinotubular junction (circled dotted arrow). $R$, Right coronary sinus; $N$, noncoronary sinus; $L$, left coronary sinus; $P$, tongue-shaped bovine pericardial patch. 
In conclusion, our novel method might effectively reconstruct aortic sinuses and annuluses simultaneously without an additional risk of intraoperative bleeding. However, the number of patients treated with this method is low, and a long-term follow-up with a significant patient cohort is mandatory to confirm its effectiveness.

\section{References}

1. Yacoub MH, Gehle P, Chandrasekaran V, Birks EJ, Child A, Radley-Smith R. Late results of a valve-preserving operation in patients with aneurysms of the ascending aorta and root. J Thorac Cardiovasc Surg. 1998;115:1080-90.

2. David TE, Ivanov J, Armstrong S, Feindel CM, Webb GD. Aortic valve-sparing operations in patients with aneurysms of the aortic root or ascending aorta. Ann Thorac Surg. 2002;74(suppl):S1758-6.

\title{
Right ventricular exclusion procedure for right ventricular failure of Ebstein's anomaly
}

\author{
Sungkyu Cho, MD, ${ }^{\text {a }}$ Woong-Han Kim, MD, PhD, ${ }^{\mathrm{a}}$ Jae Gun Kwak, MD, ${ }^{\mathrm{b}}$ and Chun Su Park, MD, ${ }^{\mathrm{a}}$ \\ Seoul, Korea
}

Ebstein's anomaly is a broad spectrum of disease characterized by varying degrees of downward displacement of the septal and posterior leaflets of the tricuspid valve into the right ventricular cavity and atrialized and dilated changes of the right ventricle. There are various surgical techniques according to the severity of desease. ${ }^{1}$ There are some descriptions about the right ventricular exclusion procedure for progressed congestive right-sided heart failure after primary biventricular repair or neonatal stenotic Ebstein's anomaly. ${ }^{2,3}$ We evaluated the preoperative and postoperative cardiac function and ventricular size by cardiac magnetic resonance imaging after the right ventricular exclusion procedure in Ebstein's anomaly.

\section{CLINICAL SUMMARY}

A 21-year-old woman who was born with Ebstein's anomaly (Carpentier type B-C) underwent tricuspid valve repair and right ventricular volume reduction with the Danielson technique at 5 years of age and tricuspid valve replacement and bidirectional cavopulmonary shunt at 16 years of age. At 21 years of age, she had severe congestive right ventricular failure (New York Heart Association

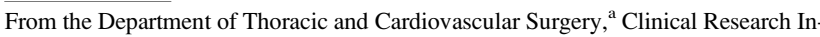
stitute, Seoul National University Children's Hospital, Seoul National University College of Medicine, Seoul, Korea; and Department of Cardiothoracic Surgery, ${ }^{b}$ Sejong General Hospital, Bucheon, Korea.

Received for publication Aug 4, 2008; accepted for publication Sept 11, 2008; available ahead of print Jan 27, 2009.

Address for reprints: Woong-Han Kim, MD, PhD, Department of Thoracic and Cardiovascular Surgery, Seoul National University College of Medicine, Seoul National University Children's Hospital, 28 Yongon-Dong, Jongno-Gu, Seoul, 110-744, Korea (E-mail: woonghan@snu.ac.kr).

J Thorac Cardiovasc Surg 2009;138:1246-8

$0022-5223 / \$ 36.00$

Copyright (C) 2009 by The American Association for Thoracic Surgery doi:10.1016/j.jtcvs.2008.09.029
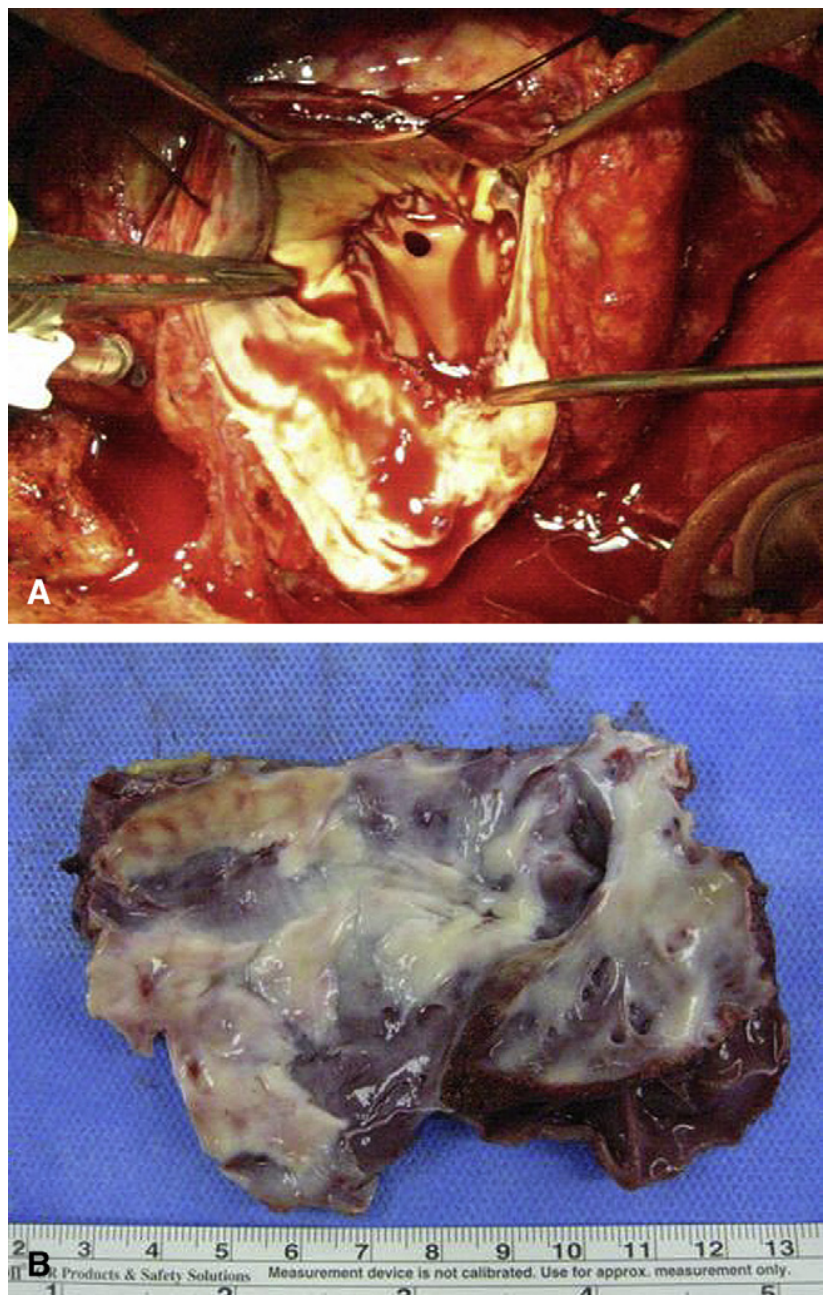

FIGURE 1. A, Previous tricuspid valve orifice was closed with a bovine pericardial patch while creating a $5-\mathrm{mm}$ atrioventricular communication. $\mathrm{B}$, Resected right ventricle. 